Meta

Journal des traducteurs

Translators' Journal

\title{
La refrancisation de l'assurance et ses conséquences
}

\section{Louis-Paul Béguin}

Volume 13, numéro 2, juin 1968

URI : https://id.erudit.org/iderudit/002019ar

DOI : https://doi.org/10.7202/002019ar

Aller au sommaire du numéro

Éditeur(s)

Les Presses de l'Université de Montréal

ISSN

0026-0452 (imprimé)

1492-1421 (numérique)

Découvrir la revue

Citer cet article

Béguin, L.-P. (1968). La refrancisation de l'assurance et ses conséquences. Meta, 13(2), 62-66. https://doi.org/10.7202/002019ar d'utilisation que vous pouvez consulter en ligne.

https://apropos.erudit.org/fr/usagers/politique-dutilisation/ 


\section{LA REFRANCISATION DE L'ASSURANCE ET SES CONSEQQUENCES}

\section{LES ÉTABLES DU ROI AUGIAS}

Pour nettoyer les étables du roi Augias, Hercule détourna le fleuve Alphée de son cours. Pour refranciser le service de traduction d'une compagnie d'assurance, faudra-t-il l'aide d'un nouvel Hercule ? Il est en effet facile de déplorer la faiblesse de la langue française dans le domaine de l'assurance; il n'est pas aussi facile d'y remédier.

Depuis quelque temps, on a pu lire dans Meta, dans les journaux et autres revues québécoises, des articles exposant d'une manière évidente les lacunes de la langue technique des contrats d'assurance au Canada. Nous, les traducteurs des compagnies d'assurance, savions que nos documents avaient été, jusqu'alors, écrits selon une "langue en travesti » comme dit M. Jean-Paul de Grandpré ${ }^{\text {. Nous }}$ étions conscients des «calques injustifiés, des anglicismes et des barbarismes». D'autre part, nous nous sommes reconnus lorsque $\mathbf{M}$. de Grandpré mentionna les efforts individuels dejà tentés « et le fait qu'un certain nombre de compagnies anglophones ont accepté une bonne partie de ce que nous pourrions appeler la nouvelle terminologie ». Car nous avons déjà commencé à nettoyer les étables du roi Augias.

Le réveil de la conscience linguistique au Québec nous donne l'occasion de prouver aux yeux de la direction anglophone le bien-fondé du nettoyage que nous voulons faire aujourd'hui du travail de nos prédécesseurs. La plus grande difficulté est de persuader la direction anglophone, de la convaincre de l'importance de la révision terminologique qui s'impose et qui n'est en somme que la pleine repossession de la langue de travail. On ne peut corriger du jour au lendemain les nombreux documents, les intercalaires, les contrats eux-mêmes, les formulaires de toutes sortes qui ont été traduits littéralement depuis des années. La direction unilingue anglaise ne comprendrait pas cette hâte. Les crédits nécessaires pour accomplir une telle cuvre ne peuvent être obtenus que si la direction est convaincue que l'intérêt

1. « Le traducteur et l'assurance sur la vie », META, vol. XI, $\mathrm{n}^{\circ} 2$, juin 1966, p. 70 , et $\mathrm{n}^{\circ} 3$, septembre 1966, p. 99 
de la compagnie est en jeu. M. Darbelnet, à ce sujet, a dit très justement: « De ce danger qu'est la traduction littérale, le traducteur doit être convaincu au point de pouvoir à son tour convaincre son employeur. ${ }^{2}$ En effet, la raison de cette réforme n'a de sens profond que pour les francophones. L'intérêt nouveau porté au fait français dans un Québec en pleine évolution nous apportant l'appui d'un dirigisme linguistique éclairé, fournit, à tous les bureaux de traduction des compagnies d'assurance et autres entreprises, l'occasion d'insister auprès des employeurs anglais pour que la refrancisation soit à l'ordre du jour.

Une fois acquis le consentement de la direction, il faut se reposer les questions fondamentales sur la traduction, avant même de fixer les règles de refrancisation. Il faut également se mettre d'accord sur les termes techniques et leur usage. Il faut enfin accepter les conséquences administratives de l'autonomie linguistique que causera cette libération de la langue, dégagée désormais du carcan de la littéralité.

\section{L'ÉTERNELLE CONCLUSION : L'IMPOSSIBILITÉ DE TRADUIRE}

M. Jaime Torres Bodet, ancien directeur général de l'Unesco, a déclaré: « La traduction est une véritable création. ${ }^{3}$ En effet, on sait depuis longtemps qu'une traduction parfaitement exacte est impossible: il faut traduire mot à mot, donc en une langue "en travesti », ou oser recréer le document en question. Le traducteur doit-il prendre l'initiative de « recréer», choisissant cette solution au lieu de se contenter d'une traduction plate, littérale, qui ne saurait être du français, mais qui serait plus proche de la pensée de l'auteur? En outre, même si le traducteur se permet cette reconstruction à partir de la langue d'arrivée, est-il certain que le résultat sera d'une intégrité à toute épreuve ?

\section{STRUCTURE ET TRADUCTION}

Les linguistes s'accordent pour dire que l'anglais et le français découpent la réalité d'une façon différente. Ces deux langues étant structurées différemment, la traduction de l'anglais au français ne peut être qu'un compromis et la langue d'arrivée peut s'en trouver sacrifiée, si l'on n'y veille. Pour obtenir un texte tenant compte des normes du français, il est donc nécessaire de « reconstruire » le sens et le style de l'original selon la structure de la langue d'arrivée. Une traduction directe et littérale ne rendra ni la beauté, ni l'élégance, ni la précision d'un texte quel qu'il soit, parce qu'une telle traduction sera soumise entièrement à la structure de la langue de départ. Il faut donc faire de la traduction oblique selon l'expression utilisée par MM. Vinay et Darbelnet 4; il faut recréer.

Ceci étant posé, il est évident qu'en littérature comme dans d'autres domaines, techniques ou commerciaux, le traducteur devra avoir le «don d'écrire».

2. « Réflexions sur la formation générale du traducteur ", MeTA, vol. XI, n 4, décembre 1966, p. 156.

3. Cité par Robert Le Bidois dans sa préface au livre de Pierre Daviault, Langage et traduction, Ottawa, Imprimeur de la Reine, 1963.

4. J.-P. Vinay et J. Darbelnet, Stylistique comparée du français et de langlais, Montréal, Beauchemin, 1966. 


\section{LE PROBLÈME DE LA REFRANCISATION} DANS LE DOMAINE DE L'ASSURANCE

En assurance, le problème est le même, et le «don d'écrire» est donc nécessaire pour traduire le contrat, qui est à la base de toute l'activité de l'industrie de l'assurance. Il s'agit de le recréer dans la réalité linguistique du français, pour obtenir une cuvre homogène, un style clair et précis. Mais en outre, la valeur technique du document français doit égaler celle de la version originale anglaise.

Comment s'y prendre par exemple pour «nettoyer» la langue française utilisée dans une compagnie d'assurance anglophone dont le siège social se trouve à Toronto et qui a son propre bureau de traduction ? Dans un milieu strictement anglophone, à 300 milles de Montréal, les communications entre le bureau de traduction et les autres services se feront, par la force des choses, presque toujours en anglais. L'objectif du service est de traduire les contrats d'assurance et leurs annexes, la correspondance, la publicité et les messages divers destinés aux assurés francophones. Tant qu'il s'agissait de «mettre » en français des phrases anglaises structurées «à l'anglaise » le bureau de traduction restait, passivement, à la merci de l'employé anglais qui réclamait une traduction en français. Ce dernier dirigeait en quelque sorte le bureau de traduction et contrôlait le travail. Il lui soumettait par exemple des pages séparées à traduire qu'il assemblait lui-même, bien qu'il ne connût pas un mot de français. La traduction d'une page de contrat, d'un paragraphe ne pouvait être que littérale puisque le traducteur, aveugle et passif (et parfois, hélas! incompétent), n'avait aucun renseignement sur l'emploi qu'on réservait à sa traduction. "Continued on next page » à la fin d'une page était fidèlement rendu par «continué à la page suivante», même s'il arrivait que la suite se trouvât au verso. (J'ai même connu un traducteur qui insistait pour mettre les virgules dans les phrases françaises à l'endroit qu'elles occupaient dans le texte anglais !) L'assemblage par des anglophones de pages en français, souvent sans qu'une lecture finale soit faite, n'a jamais donné de bons résultats, on s'en doute. Autrement dit, tout était « pensé » en anglais.

Lorsqu'il décide de refranciser le contrat, le chef du bureau de traduction se rend compte que tout son personnel doit posséder la connaissance parfaite des deux langues de travail, s'il veut s'éloigner de la traduction littérale. Comme il s'agit, à la base, d'une traduction d'ordre technique, actuariel et juridique, le traducteur du contrat devra naturellement posséder des connaissances étendues sur l'assurance anglaise et avoir étudié en détail le mécanisme et la terminologie de l'assurance tels qu'ils existent dans le monde francophone, avec les modifications régionales nécessaires. Le chef du service de traduction a besoin qu'on lui reconnaisse une autonomie et une liberté d'action nouvelles. La refrancisation n'est possible que si, n'étant plus un étranger, il participe à l'activité de la compagnie. Il devient un «spécialiste en assurance »: sa traduction ne sera plus qu'une partie de son travail. Car il est évident qu'en s'écartant du mot à mot, le traducteur devient un assureur-traducteur et que le bureau de traduction prend une forme nouvelle. (On se rappellera que, dans un article de META sur la traduction technique, M. Horguelin parlait d'ingénieurs-traducteurs employés en France ${ }^{5}$.) On réussira ainsi à atteindre l'authenticité et la précision de la langue et à

5. "La traduction technique ", META, vol. XI, n ${ }^{\circ}$ 1, mars 1966, p. 20. 
respecter l'aspect technique des documents préparés en français. Car désormais, toutes les opérations faites en français seront «pensées» (ou « repensées») en français.

La compagnie devra donner au traducteur l'autorité de faire les réformes linguistiques nécessaires, en pleine collaboration avec les bureaux anglophones, dans les domaines qui relèvent de ses fonctions. Cet esprit d'initiative devra être encouragé par la direction anglophone car certaines mesures seront prises, pour ce qui concerne le français, qui ne seront pas nécessairement envisagées par le service anglais. Un contrat devant être réimprimé en anglais tel quel devra, par exemple, être rédigé à nouveau en français à cause des changements de terminologie qu'on désire y apporter (je pense à «bénéfice » par exemple). Il apparaît donc que la refrancisation amène, par la force des choses, un surcroît de responsabilités au traducteur.

\section{LA MÉTAMORPHOSE DU BUREAU}

\section{DE TRADUCTION}

En quelque sorte, on assiste à la métamorphose du bureau de traduction, jusqu'alors replié sur lui-même, en un service d'assurance français, travaillant en étroite collaboration avec les autres services, et s'attribuant pour ce qui est du français, certaines fonctions de gestion, jusqu'alors exercées par les services anglophones.

Cela vaudra pour tous les domaines. Un traducteur publicitaire travaillera de concert avec l'agent de publicité anglais, et rédigera l'annonce, ou la brochure, ou le message radiophonique, selon les normes de la publicité française, selon les goûts du public québécois et selon la langue et la culture françaises.

\section{LA RÉORGANISATION DU SERVICE}

Au sein du service, on établira les dossiers explicatifs des régimes, on enregistrera les nouvelles expressions et leur signification, on en dressera les listes; les glossaires et les fiches terminologiques seront préparés et mis à jour au moment de la traduction de nouveaux contrats français. Chaque traducteur se spécialisera (branche ordinaire, assurance collective, assurance-maladie, etc.), gardant toute documentation soigneusement à jour pour consultation future. Il s'agira de planifier toutes les activités de traduction, de classement, de recherches, de gestion interne, etc. Faisant lui-même partie des cadres de la compagnie, le chef du service français suivra les consignes de celle-ci, mais après les avoir interprétées, quant à leur application en français; il établira, pour ses subalternes, l'équivalent français des normes envisagées au niveau directorial. S'agit-il de préparer un contrat en français, il obtiendra de l'actuaire anglophone tous les renseignements qui pourraient l'aider. D'abord traduit, le document sera préparé sous sa forme française par les diverses unités du service français. L'édition, la mise en pages, la mise au point des instructions et directives dont on aura besoin pour établir les modèles d'intercalaires et de correspondance, pour écrire les annonces publicitaires en français, etc., toutes ces opérations de gestion seront du ressort du service français. Ainsi seront évités les effets déplorables de la traduction littérale: les contrats où les mots sont français mais où la pensée anglaise domine, les phrases sans suite, les lettres traduites mais incompréhensibles, que les assurés ne peuvent 
lire sans se croire l'objet d'une mystification. D'ailleurs, l'autonomie acquise par le service français lui permettra d'adopter les normes de la correspondance commerciale française; il faudra donc retrouver, adopter et imposer ces normes et ne plus suivre les règles de la correspondance anglaise (pas de «sincèrement vôtre », etc.).

\section{DE LA TRADUCTION À LA COLLABORATION}

À la base de toute l'activité du service, il y aura bien sûr les importantes opérations de traduction. Mais le service pourra prendre également toutes les mesures nécessaires à l'établissement des communications en français avec les succursales. Il assistera les services anglophones pour les questions relatives au français. Il formera les futurs employés bilingues que ces services désireraient incorporer dans leur rang. Au lieu d'envoyer les lettres des autres services au bureau de traduction, les employés bilingues feront d'abord un stage au sein du service français et, plus tard, pourront ainsi écrire et communiquer en français avec leurs assurés. Le trafic du courrier interne de la compagnie y gagnera certainement en rapidité.

\section{VERS UN SIËGE SOCIAL BILINGUE}

Devenant un véritable centre de «l'assurance en français», le nouveau service aura abandonné l'attitude passive d'antan, en même temps que la traduction littérale. Son expansion sera graduelle et permettra la réalisation future d'un siège social vraiment bilingue. Bien que le contrat ait été d'abord rédigé en anglais, il aura acquis une identité canadienne-française en étant « recréé », par les soins du service français, en une langue authentique. Document synallagmatique, le contrat d'assurance en langue française sera donc une convention passée entre l'actuaire anglais et son collaborateur, l'assureur-traducteur, d'une part, et l'assuré, d'autre part. Ainsi, le traducteur ne sera plus le simple témoin de la convention: dorénavant il y participera activement.

\section{CONCLUSION}

Si l'on reconnaît que ces réformes prendront du temps à se réaliser, si l'on n'oublie pas qu'il est difficile de trouver des traducteurs compétents, et si l'on est prêt à donner à ces traducteurs la formation professionnelle nécessaire, il est possible de réussir cette refrancisation et d'en assumer les conséquences. Ce n'est pas non plus trop demander que d'espérer obtenir le consentement et l'appui de la compagnie d'assurance afin d'amorcer la prise de conscience linguistique à partir du bureau de traduction d'une société privée, prise de conscience qui a déjà commencé au sein des organismes gouvernementaux du Canada français. Le bureau de traduction sera ainsi libéré des chaînes qui l'avaient empêché jusqu'alors de faire un travail dans une langue juste. Métamorphosé, le service aura désormais un but qui ne sera autre que l'objectif même de la compagnie: offrir au public québécois un "produit» valable, une protection sûre et précise en ses modalités, dans une langue authentique et selon les traditions culturelles de ce public. Avec l'aide et la compréhension de la direction, les bureaux de traduction des compagnies d'assurance deviendront de véritables services d'assurance français, pour le plus grand bien des assurés et dans l'intérêt des compagnies d'assurance.

Louis-Paul Béguin 